\title{
Performance Evaluation of Manchester Encoded Input Signal FSO System Under Different Atmospheric Condition for Different Wavelength
}

\author{
Madhubala Meena $^{1}$, Sandeep Kumar Toshniwal ${ }^{2}$ \\ ${ }^{1}$ P. G. Scholar, ${ }^{2}$ Associate Professor \\ Department of Electronics \& Communication, \\ Kautilya Institute of Technology \& Engineering, Jaipur, Rajasthan, India
}

\begin{abstract}
In this paper we have analyzed free space optical communication system with input as Manchester coded signal affected by atmospheric attenuation for different values of wavelength. FSO link is analyzed $500 \mathrm{~m}$ range, $20 \mathrm{~cm}$ receiver diameter and $15 \mathrm{~mW}$ input power for different values of wavelength $(1491 \mathrm{~nm}$, $1531 \mathrm{~nm}$ and $1550 \mathrm{~nm}$ ). Values of attenuation are $5 \mathrm{~dB} / \mathrm{Km}, 20 \mathrm{~dB} / \mathrm{Km}, 40 \mathrm{~dB} / \mathrm{Km}$ and $70 \mathrm{~dB} / \mathrm{Km}$. Qfactor and eye height are calculated for each case. Eye diagrams are generated to produce the desired results using OptiSystem tool.
\end{abstract}

Key Words: FSO, Attenuation, Manchester Signal, Eye Height, Q Factor

\section{INTRODUCTION}

The need of advanced bandwidth is growing these days. Very high data rate communication is required that can be presented by FSO and can exchange RF communication in various applications. RF can offer data rate of upto many Mbps, but there is a restriction of spectrum congestion, interference and issues connected to license. FSO is a efficient as well as high bandwidth access technique, which has accepted growing cerebration with modern development of the application. New techniques to decrease the restriction of FSO require to be investigated in close future to take the peak advantage of wireless optical link. The FSO also provide options for ground to satellite link, inter-satellite link along with the terrestrial link. Highly accurate tracking system is still needed to observe the optical beam to cut the geometrical fading in FSO links which in turn gains the link interval.
Many techniques are being looked into by several investigators to degrade the tracking trouble. The tracking issue gets more terrible in case of ground to satellite links and inter-satellite link.

Free Space Optics (FSO) technology-supported wireless systems are not without inquiring. These systems are capable to atmospherical status which insert errors and can create the system unusable for few time-periods. The decrease in free space optical link is mostly due to attention due to water haze, distribution due to water droplets (rain, snow, fog etc) as well as meteorological turbulence like glitter and beam expanding. Bit error rate execution of a FSO link and visibility range is unfavorably moved due to these. The meteorological turbulence is the major demolition over FSO links, which results in variations of the acquired signal, badly debasing the execution of FSO link. Hence, environment reduces the signal in FSO link in many paths that combine absorption, diffusion, and scintillation.

The turbulence effects can be reduced by the use of different modulation plans like as binary phase shift keying (BPSK) subcarrier intensity modulation; moreover it will be at the costs of the power efficiencies and bandwidth. By using On-Off Keying (OOK) intensity Modulation (IM) pattern is the easiest and broadly approved signalling scheme in FSO communication systems because of the simple execution. In FSO communication several deals has been completed in the adjusting and growing various coding and modulation approach. 
International Journal of Trend in Scientific Research and Development (IJTSRD) ISSN: 2456-6470

\section{MANCHESTER ENCODING AND DECODING}

Manchester encoded signal is a useful signal waveform as it exhibits the effects of level unresponsive and self- clocking. Absence of signal can be efficiently resoluted as the encoded data always expresses minimum one transition per bit. Dr. G. E. Thomas declared the concept that logic ' 0 ' can be indicated by a positive propagating signal and logic ' 1 ' can be denoted by a negative propagating signal depending on IEEE 802.4 standards in 1948. Manchester encoding technique always performs transition in waveform at centre place of data bit cycle. flow of logic ' 1 ' or ' 0 ' in NRZ waveform produces low to high transition or high to low transition individually at boundary of data bit cycle.

It does not produce big lines of logic ' 1 ' or logic ' 0 ' value. There is a proficiency of AC coupling of encrypted waveform as this approach does not need transmission of DC value. Logic ' 0 ' chased by a logic ' 0 ' indicates the absence of original NRZ data signal and Logic ' 1 ' followed by a logic ' 1 ' shows the dispute in fresh signal. Signal power is free from the data type. Reset signal is not needed in this technique. It is a very good technique to use on single core devices such as optical fiber, coaxial cable, Ethernet and Local area networks (LANs).

Manchester encoder deals as edge triggered D flip flop and also perform as an XNOR gate as shown in Fig. 1 whose truth table is given in Table 1 and waveforms for this encoding scheme are shown in Fig. 2.

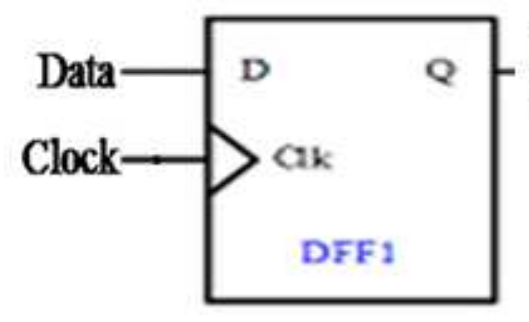

(a)

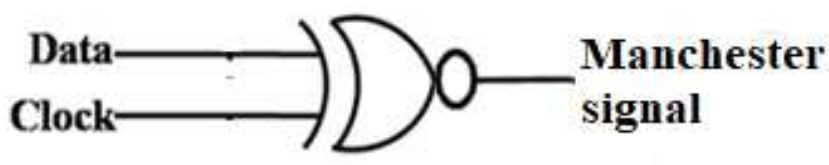

(b)

\section{Manchester signal}

(1)

Example: Suppose Input NRZ data is $(11111100001100)_{2}$ and clock is $(10101010101010)_{2}$ so XOR output will be $(01010110100110)_{2}$ and XNOR output will be $(10101001011001)_{2}$. This example is represented in Fig. 2.

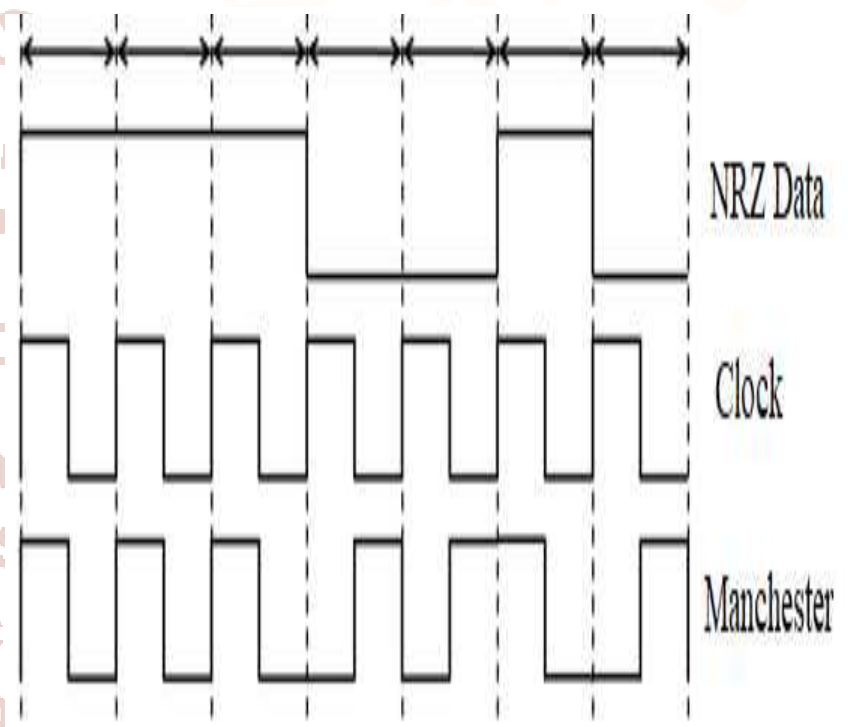

Fig. 2: Waveforms for Manchester encoding scheme

Manchester encoded signal can be decoded by passing it to XNOR gate with the same clock input. Fig. 3 shows decoder diagram and Fig. 4 shows waveforms for decoding scheme.

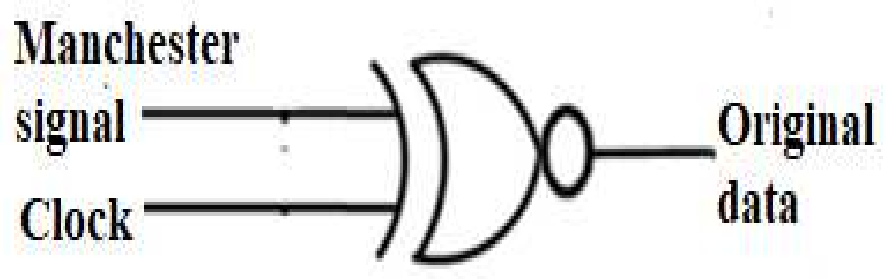

Fig. 3: Manchester decoder

Decoding example: From previous example Manchester signal is (10101001011001) $)_{2}$ and clock is $(10101010101010)_{2}$ so XOR output will be $(00000011110011)_{2}$ and XNOR output will be $(11111100001100)_{2}$ which represents the original NRZ data as shown in Fig. 4.

Fig-1 Manchester encoder (a) D-flip flop (b) XNOR gate 
International Journal of Trend in Scientific Research and Development (IJTSRD) ISSN: 2456-6470

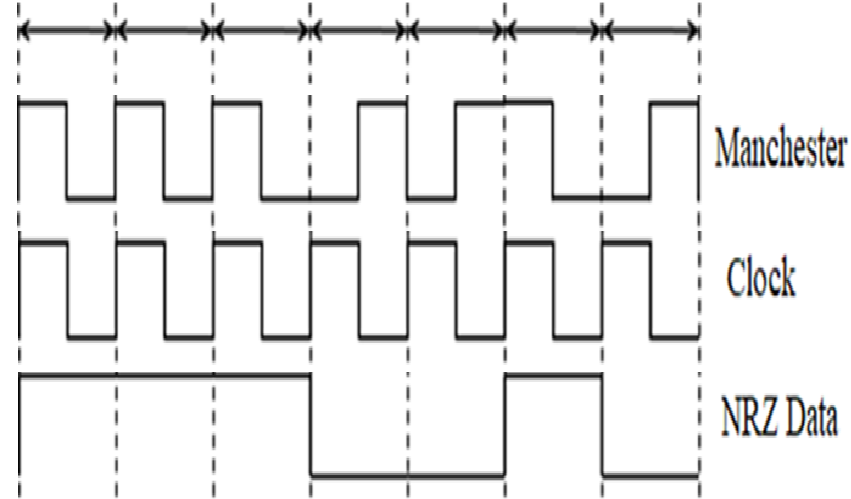

Fig. 4: Waveforms for Manchester decoding scheme

\section{PROPOSED DESIGN}

In Fig.5 initially a user defined bit sequence generator having $15 \mathrm{Gbps}$ data rate is used to generate user defined bit pattern also called data (110011). One more user defined bit sequence generator having 15 Gbps data rate is implemented to generate a clock signal (101010).
Then data and clock signals are passed through binary XOR component to produce binary output. Now this output is passed through binary NOT gate to produce binary value of Manchester signal. This Manchester signal is then converted into electrical signal through NRZ pulse generator. Then this NRZ pulse is modulated with CW laser source by MZM (MachZehnder modulator). This modulator converts the pulse into optical signal. Optical signal is then amplified through EDFA.

Then it is transmitted into atmosphere through FSO channel. This part of system completes the transmitter section of FSO system. Now at receiver end optical signal is received and amplified through optical amplifier. Received optical signal is passed through Avalanche photodetector and gets converted into electrical signal.

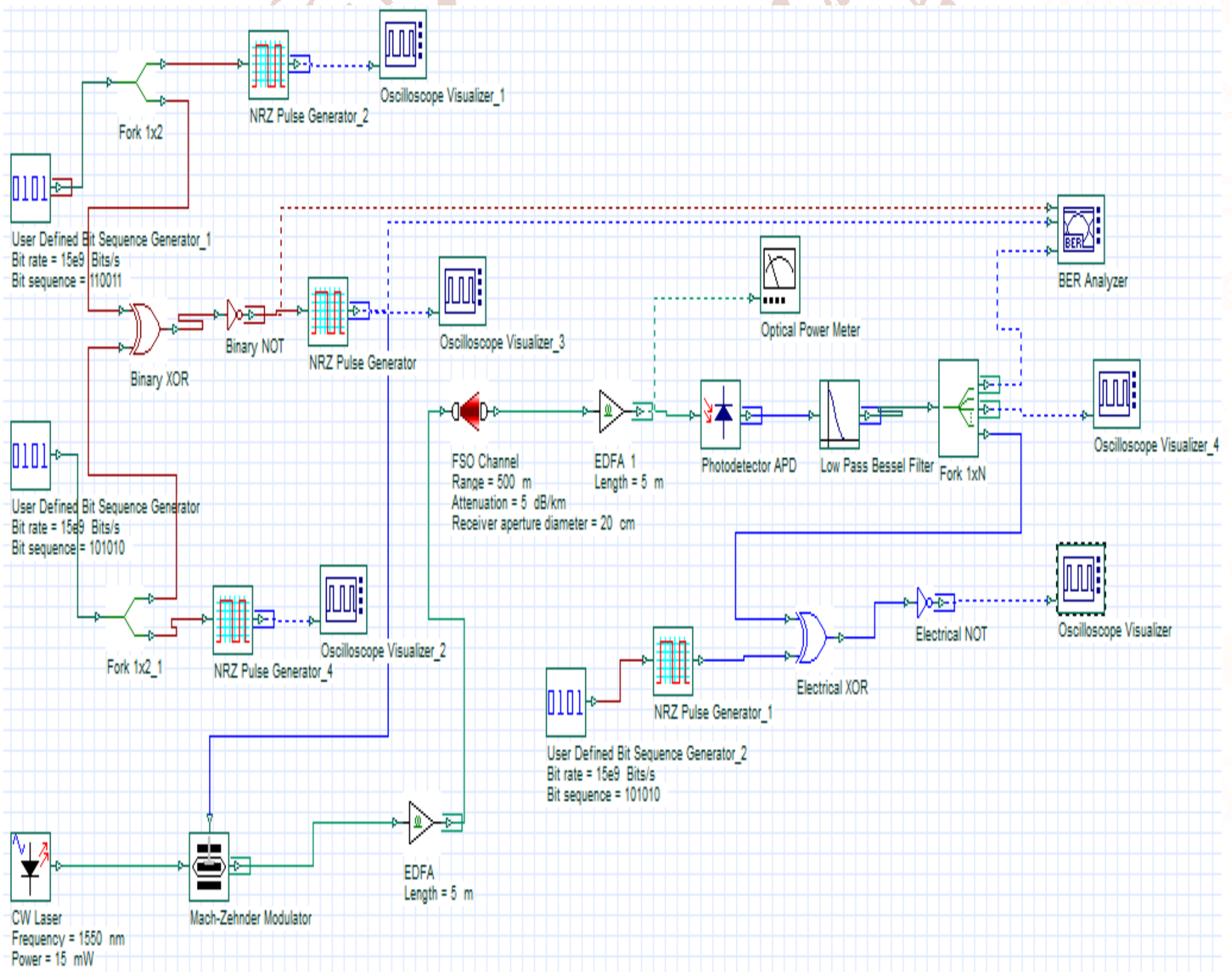

Fig-5: FSO system with Manchester Encoding 
Now the electrical signal is passed through low pass Bessel filter to eliminate unwanted high frequency noise signals. Here we obtain our Manchester encoded signal. This signal is transmitted to BER analyzer to calculate properties Q-factor, eye height and to one input of electrical XOR gate of decoder circuitry. On the second input of XOR gate, electrical pulse of clock signal is provided. Output signal is passed through electrical NOT gate where we obtain decoded signal which can be seen by oscilloscope visualizer. Fork $1 \times 2$ makes copy of the input signal.

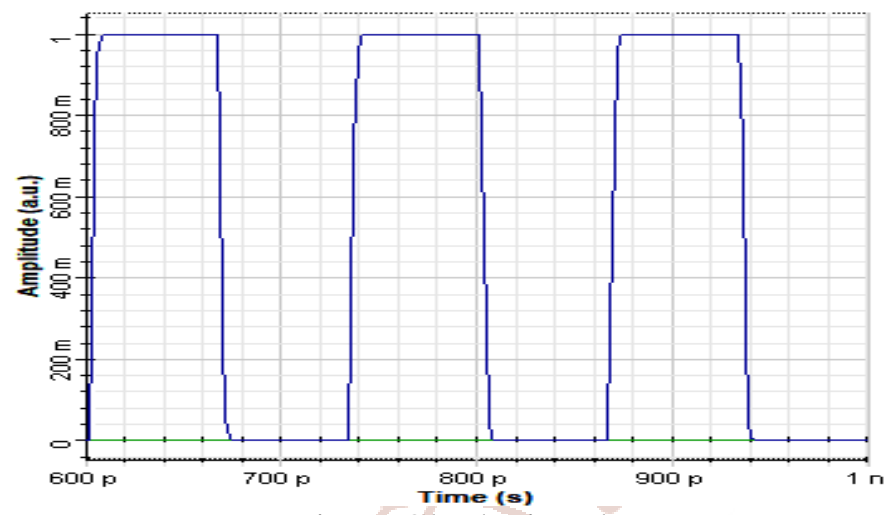

Fig. 6 Clock signal

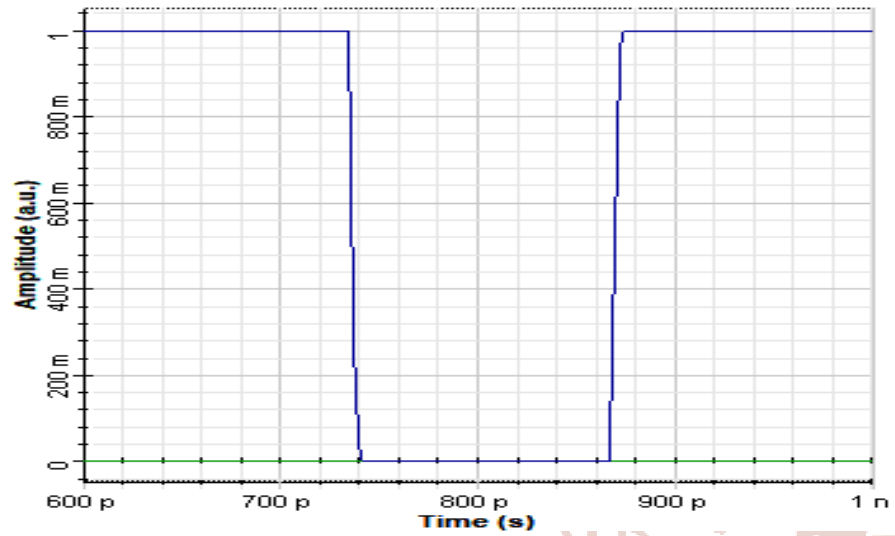

Fig. 7 Input data signal

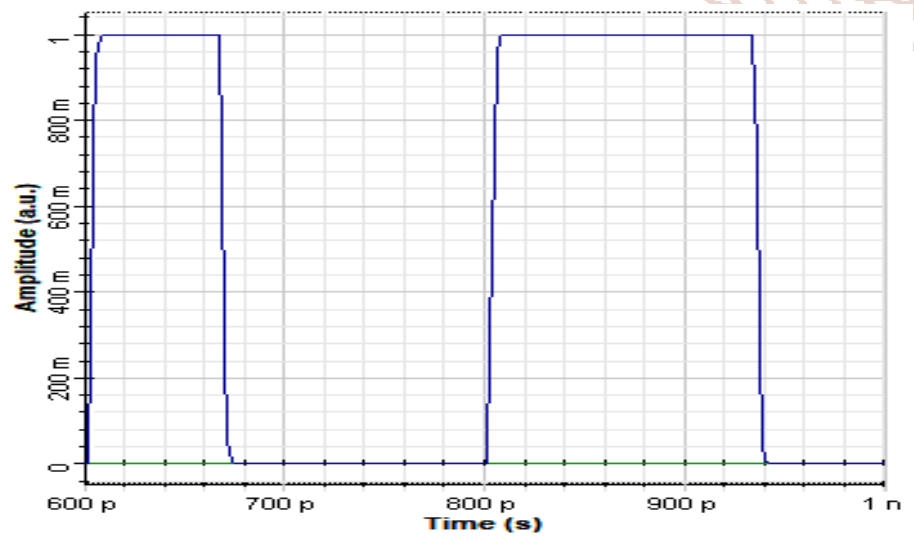

Fig. 8 Transmitted Manchester signal

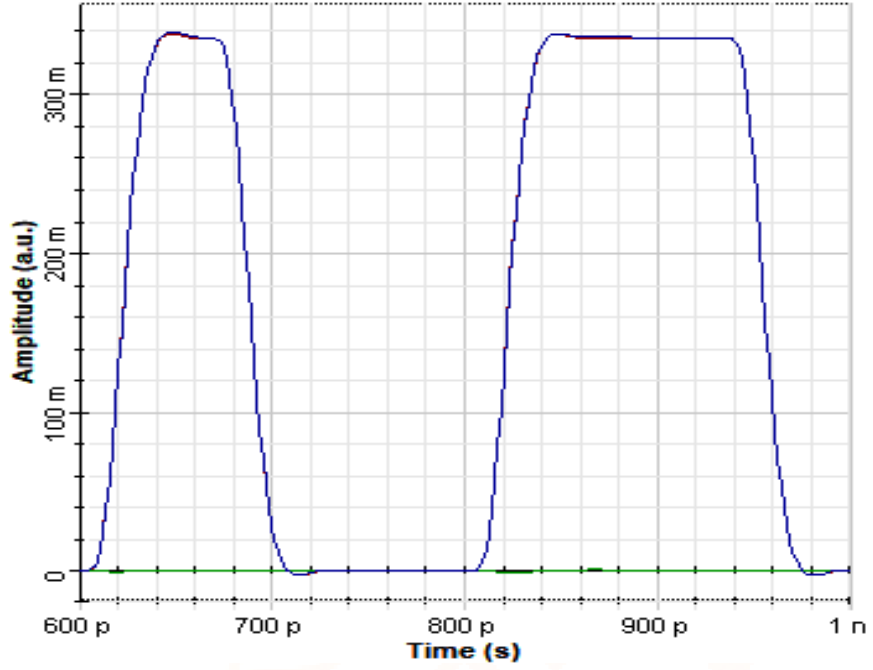

Fig. 9 Received Manchester signal

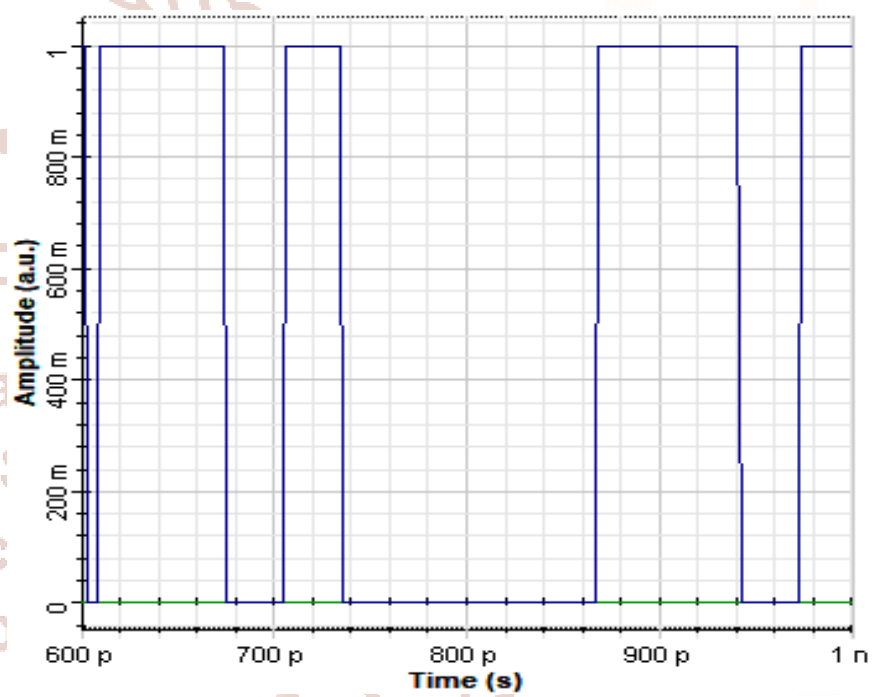

Fig. 10 Decoded data signal

\section{SIMULATION RESULTS}

All FSO systems are simulated at $15 \mathrm{Gbps}$ data rate to determine the Q-factor and eye height for different values of wavelength under different values of attenuation. In this analysis range is kept constant at $500 \mathrm{~m}$ receiver diameter at $20 \mathrm{~cm}$. Results for different wavelengths under different climatic conditions are shown below:

Table 2 Simulation results at $1491 \mathrm{~nm}$ wavelength Input power $=15 \mathrm{~mW}$, Range $=500 \mathrm{~m}$, Receiver diameter $=20 \mathrm{~cm}$

\begin{tabular}{|c|c|c|}
\hline Attenuation $(\mathbf{d B} / \mathbf{K m})$ & Q-factor & Eye Height \\
\hline 5 & 267.38 & 0.0047 \\
\hline 20 & 145.76 & 0.0016 \\
\hline 40 & 63.77 & 0.0003 \\
\hline 70 & 7.82 & 0.0019 \\
\hline
\end{tabular}


International Journal of Trend in Scientific Research and Development (IJTSRD) ISSN: 2456-6470

Table 3 Simulation results at $1531 \mathrm{~nm}$ wavelength Input power $=15 \mathrm{~mW}$, Range $=500 \mathrm{~m}$, Receiver diameter $=20 \mathrm{~cm}$

\begin{tabular}{|c|c|c|}
\hline Attenuation (dB/Km) & Q-factor & Eye Height \\
\hline 5 & 400.23 & 0.334 \\
\hline 20 & 214.83 & 0.314 \\
\hline 40 & 68.48 & 0.269 \\
\hline 70 & 8.67 & 0.068 \\
\hline
\end{tabular}

Table 4 Simulation results at $1550 \mathrm{~nm}$ wavelength Input power $=15 \mathrm{~mW}$, Range $=500 \mathrm{~m}$, Receiver diameter $=20 \mathrm{~cm}$

\begin{tabular}{|c|c|c|}
\hline Attenuation $(\mathbf{d B} / \mathrm{Km})$ & Q-factor & Eye Height \\
\hline 5 & 420.69 & 0.333 \\
\hline 20 & 194.14 & 0.304 \\
\hline 40 & 63.22 & 0.194 \\
\hline 70 & 9.41 & 0.011 \\
\hline
\end{tabular}

Graphical representation of Table 2, 3 and 4 are shown below:

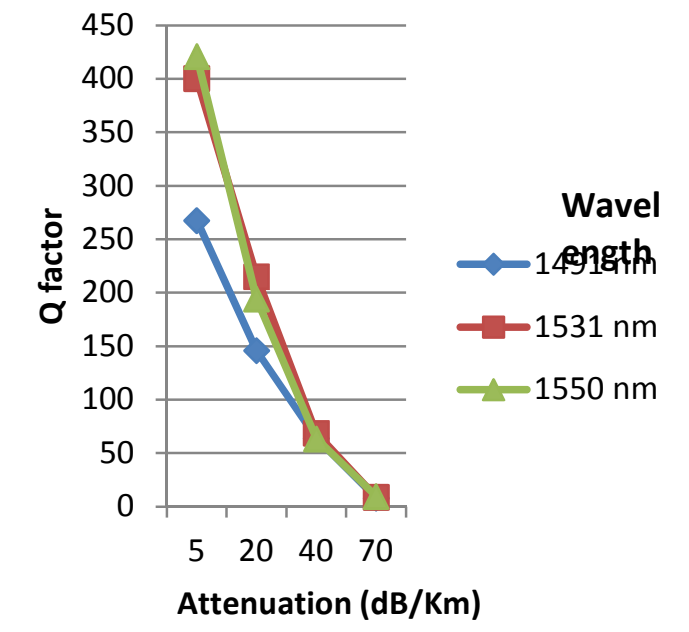

Fig. 11 Q factor comparison for different wavelength

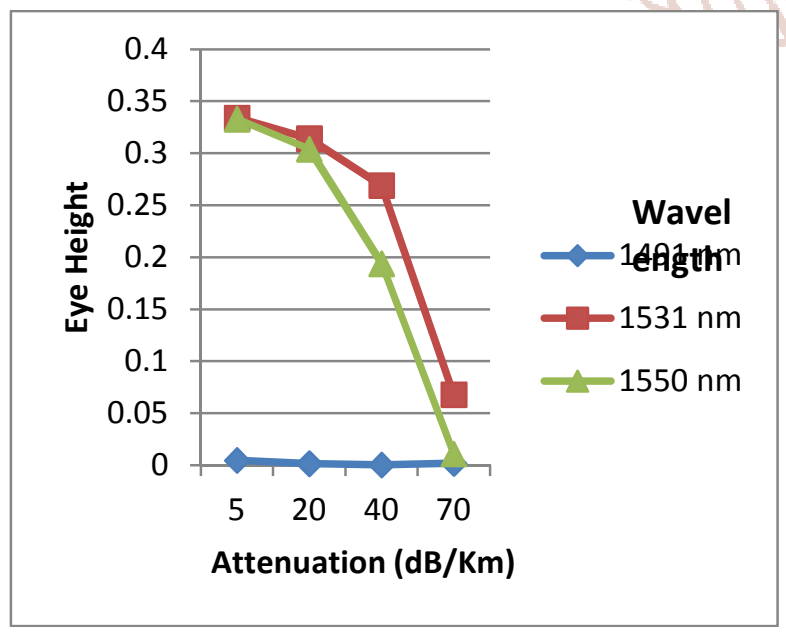

Fig. 12 Eye height comparison for different wavelength

\section{CONCLUSIONS}

FSO applications, with their strength in producing high data rate and price effective optical communications, are among the most efficient and promising methods to eliminate the limitations of contemporary communication devices and fulfill the highly increasing demand for higher speed information sharing. FSO communication system is designed with Manchester encoded signal as an input signal. This system is analyzed under different values of atmospheric attenuation such as $5 \mathrm{~dB} / \mathrm{Km}, 20$ $\mathrm{dB} / \mathrm{Km}, 40 \mathrm{~dB} / \mathrm{Km}$ and $70 \mathrm{~dB} / \mathrm{Km}$. FSO system is simulated at all attenuation factors for different wavelength.

\section{REFERENCES}

1. Arun K. Majumdar and Jennifer C. Ricklin, "FreeSpace Laser Communications: Principles and Advances", Springer, 2008.

2. Hennes Henniger and Otakar Wilfert, "An introduction to free-space optical communications", Journal of Radio Engineering, 2010

3. Ateve Hranilovic, "Wireless Optical Communication Systems", Springer, eBook ISBN: 0-387-22785-7, 2005.

4. H. Hemmati, "Interplanetary laser communications", Optics and Photonics News, Vol. 18, pp. 22-27, Nov. 2007.

5. S. Zoran, F. Bernhard and L. Hanspeter, "Freespace laser communication activities in Europe: SILEX and beyond", IEEE Lasers and ElectroOptics Society (LEOS), $19^{\text {th }}$ Annual Meeting, 2006.

6. Z. Sodnik, B. Furch and H. Lutz, "Free-space laser communication activities in Europe: SILEX and beyond", $19^{\text {th }}$ Annual Meeting of the IEEE Lasers and Electro-Optics Society 2006.

7. R. Lange and B. Smutny, "Homodyne BPSKbased optical inter-satellite communication Links", In Proceedings of the SPIE, Free-Space Laser Communication Technologies, San Jose (USA), 2007.

8. G. Hansel and E. Kube, "Simulation in the Design Process of Free Space Optical Transmission Systems", Proc. $6^{\text {th }}$ Workshop, Optics in Computing Technology, Paderborn (Germany), 2003.

9. S. Mohammad Navidpour, Murat Uysal and Mohsen Kavehrad, "BER Performance of FreeSpace Optical Transmission with Spatial Diversity", IEEE Transactions on Wireless Communications, 2007. 